\title{
Preliminary application of 3.0 T magnetic resonance chemical exchange saturation transfer imaging in brain metastasis of lung cancer
}

Yonggui Yang ${ }^{1}$, Xiaobo Qu ${ }^{2}$, Yihui Huang ${ }^{2}$, Khan Afsar ${ }^{2}$, Gen Yan ${ }^{1}$, Gang Guo ${ }^{1 *}$ and Shaoyin Duan ${ }^{3^{*}}$

\begin{abstract}
Background: Lung cancer brain metastases are very common and one of the common causes of treatment failure. We aimed to examine the clinical use of chemical exchange saturation transfer (CEST) technology in the evaluation of brain metastases for lung cancer diagnosis and prognosis.

Methods: We included26 cases of lung cancer brain metastases, 15 cases of gliomas, and 20 cases with normal tests. The magnetization transfer ratio (MTR;3.5 ppm) image from the GRE-EPI-CEST sequence was analyzed using the ASSET technique and APT technology. The MTR values were measured in the lesion-parenchymal, edema, and non-focus regions, and the MTR image was compared with the conventional MRI. ANOVA and t-test were used for statistical analysis.

Results: The lesion-parenchymal, edema, and non-focus areas in the metastatic-tumor-group were red-yellow, yellow-green, and green-blue, and the MTR values were $3.29 \pm 1.14 \%, 1.28 \pm 0.36 \%$,and $1.26 \pm 0.31 \%$, respectively. However, in the glioma-group, the corresponding areas were red, red-yellow, and green-blue, and the MTR values were $6.29 \pm 1.58 \%, 2.87 \pm 0.65 \%$, and $1.03 \pm 0.30 \%$, respectively. The MTR values of the corresponding areas in the normal-group were $1.07 \pm 0.22 \%, 1.04 \pm 0.23 \%$, and $1.06 \pm 0.24 \%$, respectively. Traditional MR images are in blackwhite contrast and no metabolic information is displayed.

The MTRvalues of the three regions were significantly different among the three groups. The values were also significantly different between the parenchymal and edema areas in the metastatic-tumor-group. There were significant differences in the MTR values between the non-lesion and edema regions, but there was no significant difference between the edema and non-focus areas. In the glioma-group, there were significant differences in the MTR values between the parenchymal and edema areas, between the parenchymal and non-focus areas, and between the edema and non-focus areas.
\end{abstract}

Conclusions: CEST reflects the protein metabolism; therefore, early diagnosis of brain metastases and assessment of the prognosis can be achieved using molecular imaging.

Keywords: Magnetic resonance imaging, Chemical exchange saturation transfer, Metastases, Magnetization transfer ratio

\footnotetext{
* Correspondence: guogangxm@163.com; xmdsy@xmu.edu.cn

${ }^{1}$ Department of Radiology, The Second Affiliated Hospital of Xiamen Medical

College, Xiamen, Fujian Province, China

${ }^{3}$ Department of Radiology, Zhongshan Hospital Xiamen University, Xiamen,

Fujian Province, China

Full list of author information is available at the end of the article
}

(c) The Author(s). 2020 Open Access This article is distributed under the terms of the Creative Commons Attribution 4.0 International License (http://creativecommons.org/licenses/by/4.0/), which permits unrestricted use, distribution, and reproduction in any medium, provided you give appropriate credit to the original author(s) and the source, provide a link to the Creative Commons license, and indicate if changes were made. The Creative Commons Public Domain Dedication waiver (http://creativecommons.org/publicdomain/zero/1.0/) applies to the data made available in this article, unless otherwise stated. 


\section{Background}

Metabolic equilibrium and acid-base balance are important components for preserving the body's homeostasis [1]. The shifts in intracellular and extracellular metabolites and $\mathrm{pH}$ values in the early stages of the diseasehave received great attention. As an important branch of magnetic resonance imaging (MRI), chemical exchange saturation transfer (CEST) has the advantages of nonradiative, non-invasive, selective $T_{1}$ and $T_{2}$ contrast and metabolic imaging $[2,3]$, extending MRI from conventional anatomical imaging to live metabolic imaging, $\mathrm{pH}$ imaging, and other subtypes. It extends the new field of MRI molecular imaging, providing a new means of disease diagnosis, treatment, and even prevention.

Brain metastases of lung cancer are very common and serious and are also one of the common causes of treatment failure $[4,5]$. Tracking metabolite changes during brain metastases is a problem, and CEST provides a new approach for solving it. We aimed to explore the clinical use of 3.0 T MRI CEST technology in the diagnosis and prognosis evaluation of lung cancer brain metastases.

\section{Methods}

\section{General information}

From January to July 2018, 26 patients with lung cancer brain metastases (17 males and 9 females, with a mean age 51.36 [48-73]years), 15 patients with gliomas (10 males and 5 females, with a mean age 43.20 [3848]years), and 20 normal controls (14 males and 6 females,with a mean age 25.87 [24-29] years) were enrolled. The primary tumor of the 26 patients with brain metastases was lung cancer, including 8 cases of adenocarcinoma, 4 cases of small cell lung cancer, 11 cases of squamous cell carcinoma, and 3 cases of large cell lung cancer.

This study was reviewed and approved by the Medical Ethics Committee of Xiamen Medical College's Second Affiliated Hospital (Approval No: 2014004). All participants provided written informed consent.

\section{Imaging method}

Routine MRI examination (T1WI, T2WI, T2 FLAIR), enhanced analysis(T1WI $+C)$, diffusion-weighted imaging (DWI), susceptibility-weighted imaging (SWI), arterial spin labeling (ASL), and CEST were obtained using Discovery MR750W 3.0 T(GE Medical Systems LLC, WI USA) .

The CEST imaging parameters were as follows. Oblique axis (OAx) CEST imaging: gradient recalled echoecho planar imaging (GRE-EPI) CEST sequence using array spatial sensitivity encoding technique (ASSET), $\mathrm{NEX}=1, \quad \mathrm{TR}=2000 \mathrm{~ms}, \quad \mathrm{TE}=$ minimum, number of shots $=1$, flip angle $=20^{\circ}, \mathrm{FOV}=24 \mathrm{~cm} \times 24 \mathrm{~cm}$, matrix $=$ $128 \times 128, \quad$ frequency $=128, \quad$ phase $=128, \quad$ frequency direction $=\mathrm{R} / \mathrm{L}$, slice thickness $=4 \mathrm{~mm}$, spacing $=0 \mathrm{~mm}$, number of slices $=1$, acquisition time: $1 \min 44 \mathrm{~s}$.

CEST saturation parameters: Continuous saturation, Amplitude of saturtion RF $=2$ ut, Duration of saturtion $\mathrm{RF}=400 \mathrm{~ms}$, Number of saturtion RF $=4$, Crusher amplitude scale $=1$,Extra space after crusher $=0 \mathrm{~ms}$.

\section{Inclusion and exclusion criteria Inclusion criteria}

All patients had clinically and pathologically confirmed findings and available MRI results and other related data.

Two high-grade imaging physicians with more than 10 years of experience in imaging diagnostics and more than 3 years of experience as attending physiciansparticipated in the inclusion process. The above data was combined with MRI, including metastatic or intracranial primary tumors, lesion location, number, size, morphology, imaging diagnosis and differential analysis of tumor signal manifestations, lesion enhancement, and edema around the tumor. The blood supply and bleeding of the tumor were also observed on SWI images. Both doctors read the films individually, and the consistent summary opinions were confirmed as true.

Patients who were reliably reported to be healthy were included in the normal group, those with lung cancer brain metastases were included in the metastatic tumor group, and those with high-grade gliomas were included in the glioma group.

\section{Exclusion criteria}

The following were the exclusion criteria: 1 ) the imaging data did not meet the diagnostic requirements; 2) lesions less than $1 \mathrm{~cm}$, or 3) intra tumor bleeding on SWI.

\section{Data processing and analysis}

CEST post-process was done using the ADW4.6 Workstation Function tool 2.0 Research version APT software. The observed $3.5 \mathrm{ppm}$ magnetization transfer rate (MTR) map was used to quantify the MTR value of the lesion parenchymal region, edema area, and non-focal area, measured in percentages, compared to the plain scan, enhancement, DWI, SWI, and ASL images of the 3.0 T MRI instrument.

While drawing the area of interest, we referenced the dominant signal on the enhanced and DWI images, to avoid cysts and necrotic zones. The lesion parenchymal area was selected to be the most prominent area of the lesion or the lower ADC value of the DWI. The edema area was selected in the edema zone within $2 \mathrm{~cm}$ of the tumor in conjunction with T2 FLAIR and enhancement. In the normal white matter region on the contra lateral side, the non-lesion area was selected. Both areas were 
selected to reduce the blood supply to vascular areas and cerebrospinal fluid areas as much as possible.

The measured data were expressed as mean \pm standard deviation and statistical analysis was performed using SPSS 23.0 from IBM. The MTR values of the focal parenchyma, edema, and the non-lesion areas, each for the normal, metastatic tumor, and the glioma groups, and the corresponding areas between the groups were first analyzed by one-way ANOVA with test criterion $\alpha=$ 0.05 . When the one-way ANOVA analysis of variance was $P<0.05$, the independent $\mathrm{t}$-test experiment was compared with the mean number of samples. $\mathrm{P}<0.05$ was considered statistically significant with the test standard $\alpha=0.05$.

\section{Results}

On the MTR map, the focal parenchymal areas, the edema areas, and the non-lesion areas of the metastatic tumor group were reddish yellow, yellowish green, and greenish blue, respectively, and the MTR values were $3.29 \pm 1.14 \%, 1.28 \pm 0.36 \%$, and $1.26 \pm 0.31 \%$, respectively. These areas of the glioma group were red, reddish yellow, and greenish blue, and the boundary between the parenchyma and the edema was unclear. The corresponding MTR values were $6.29 \pm 1.58 \%, 2.87 \pm$ $0.65 \%$, and $1.03 \pm 0.30 \%$. The same areas of the normal group were greenish blue, and the MTR values were $1.07 \pm 0.22 \%, 1.04 \pm 0.23 \%$, and $1.06 \pm 0.24 \%$. Traditional MRI images are in black and white contrast with no metabolic information (Figs. 1, 2, 3, and Table 1).

The results of the one-way ANOVA analysis of variance showed that the MTR values in the focal parenchymal areas, edema areas, and the non-lesion areas of the normal group were $\mathrm{F}=0.074, P=0.929>0.05$, no statistical difference. The MTR values in the corresponding areas of different groups and between different groups were significantly different $(P<0.05$ for all).

In the metastatic tumor and normal groups $(\mathrm{t}=-$ $8.550,-2.994,-2.403, P=0.000,0.024,0.021)$ and the glioma group $(\mathrm{t}=-6.437,-8.399,2.382, P=0.000$, $0.000,0.024)$, the corresponding MTR values of the corresponding three regions were statistically different $(P<$ $0.05)$, as shown in Table 2 . The MTR values in the focal parenchymal areas of the metastatic tumor group were compared with those of the edema areas $(\mathrm{t}=7.207, P=$ $0.000)$ and the non-lesion areas $(t=8.762, P=0.000)$ and were all statistically different $(\mathrm{P}<0.05)$. The MTR values in the edema areas and the non-lesion areas were

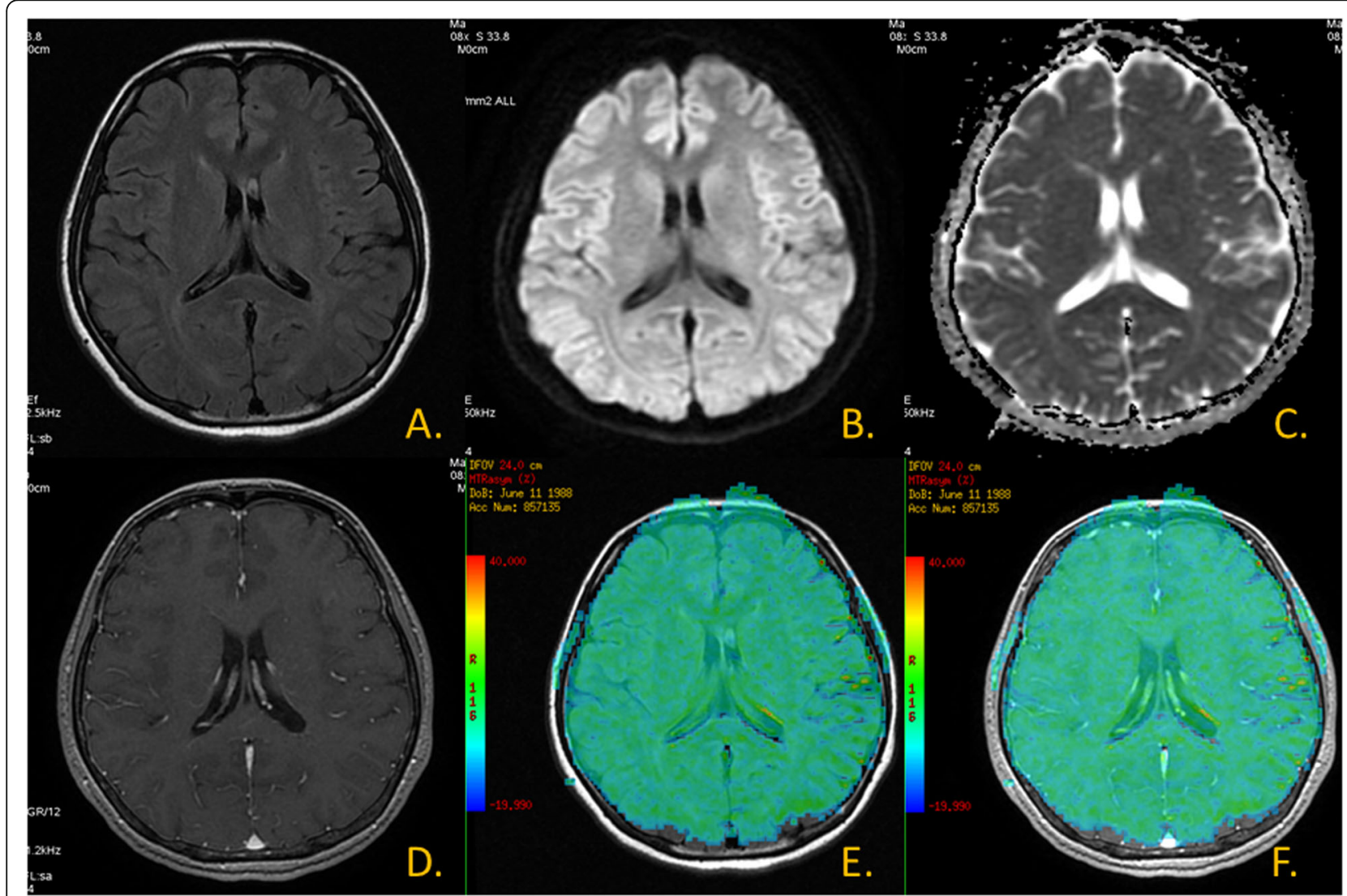

Fig. 1 Normal volunteers. a.T2 FLAIR image; b.DWl; c.ADC map; d.T1WI + C; e. CEST MTR map and T2 FLAIR image fusion; f. CEST MTR map and $\mathrm{T} 1 \mathrm{WI}+\mathrm{C}$ fusion 


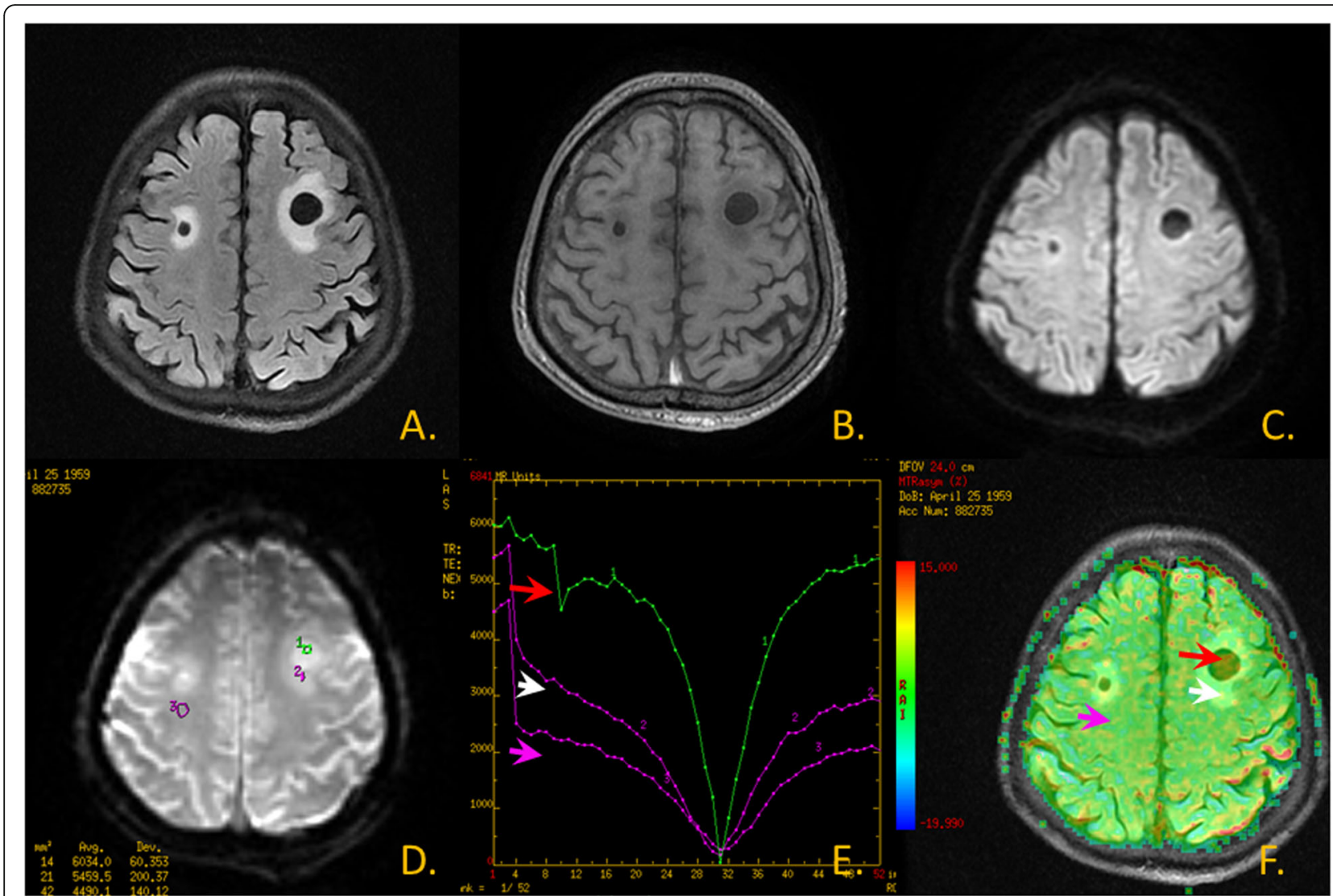

Fig. 2 Metastatic tumor images. a.T2 FLAIR image; b.T1Wl; c. DWl; d. CEST Original map; e. CEST Z spectrum: The red arrow area is shown as lesion-parenchymal areas, The white arrow area is shown as edema areas and The pink arrow area is shown as non-focus areas; $\mathbf{f}$. CEST MTR map and T2 FLAIR image fusion: The red arrow area is shown as lesion-parenchymal areas, The white arrow area is shown as edema areas and The pink arrow area is shown as non-focus areas

compared, $\mathrm{t}=0.188, P=0.852$, and there was no statistical difference $(P>0.05)$. The MTR values in the focal parenchymal areas of the glioma group were compared with those in the edema areas $(\mathrm{t}=7.748, P=0.000)$ and in the non-lesion areas $(\mathrm{t}=12.672, P=0.000)$, and all were statistically different $(P<0.05)$. The MTR values in the edema areas and the non-lesion areas were compared, $\mathrm{t}=9.926, P=0.000$, and there was a statistical difference $(P<0.05)$, as shown in Table 3.

\section{Discussion}

\section{Combination of multiple MRI techniques for brain} metastases

Traditional MR diagnosis of brain metastases is performed by routine MRI and enhanced examination, and signs of brain metastases and edema around the tumor are observed. The MRI shows that the brain metastases are slightly low or low signal intensity on $\mathrm{T}_{1} \mathrm{WI}$, high signal intensity on $\mathrm{T}_{2} \mathrm{WI}$, equal or slightly low signal intensity on $\mathrm{T}_{1} \mathrm{WI}$, and high signal intensity on $\mathrm{T}_{2} \mathrm{WI}$ in the edema regions around the tumor. The reason is that tumor cells have a relatively high water content than that of the normal white matter. Using MR-enhanced scans, brain metastases are distinguished using uniform enhancement, irregular enhancement, nodular or annular enhancement, and more specifically, partially hidden lesions. The edemas around the tumor are not enhanced. If intratumoral hemorrhage of the brain metastases is exacerbated by hypertension, some lesions show a high or moderately low $\mathrm{T}_{1} \mathrm{WI}$ signal.

The DWI and its ADC signals were used to observe the edema of lung cancer and its underlying edema. When there was a high DWI signal and decreased ADC value it reflected changes in the microscopic structure of the brain metastasis and showed other characteristics of the surrounding area of the tumor. This can effectively improve the accuracy of diagnosis and differential diagnosis of lung cancer brain metastases. This is well validated in this dataset [6].

SWI revealed tumor and peripheral blood supply, intratumoral hemorrhage, non-invasive detection of variations in magnetic sensitivity between tissues, reflecting blood oxygen levels in tissues. In this group of data, 26 cases of brain metastases and 15 cases of gliomas, SWI 


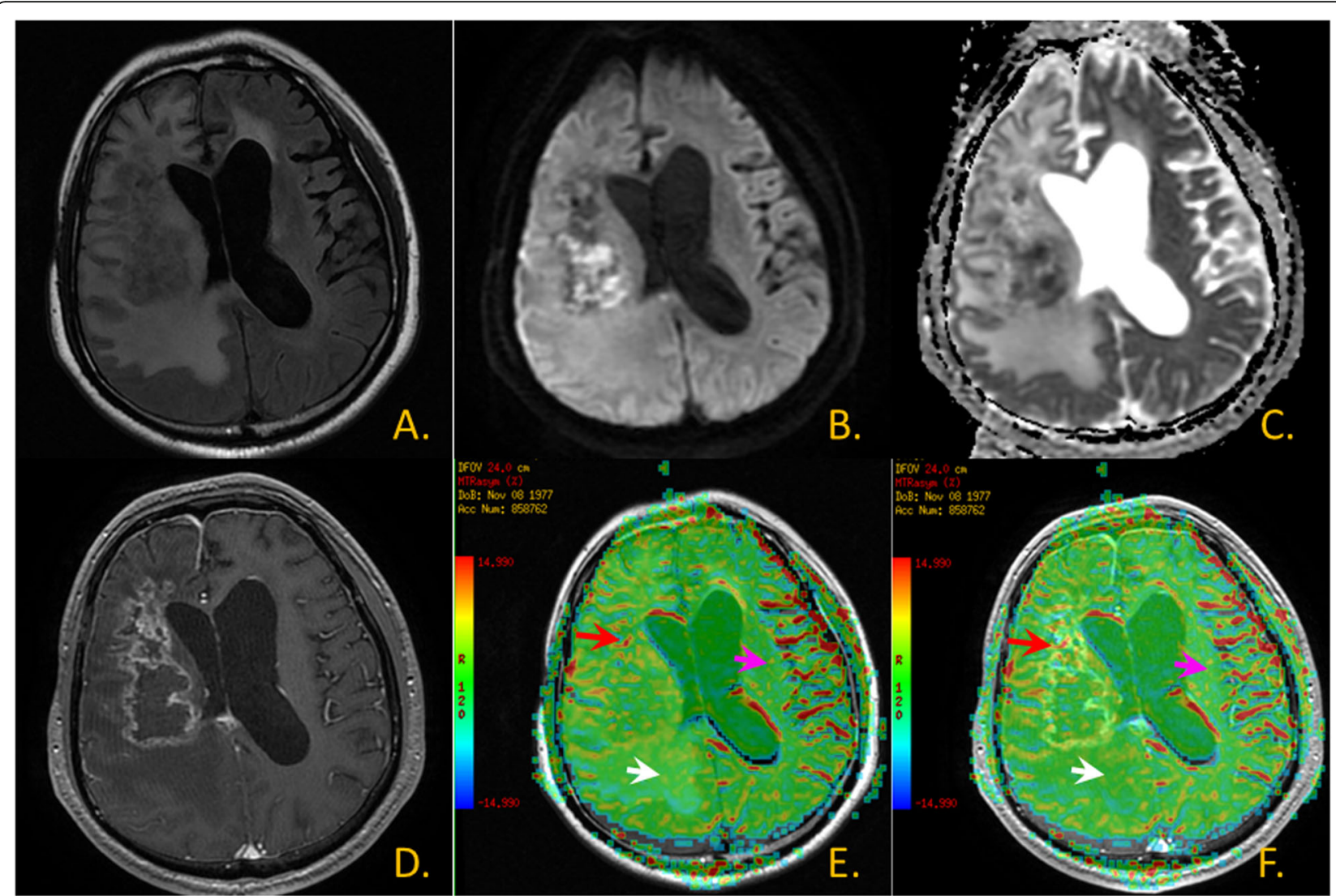

Fig. 3 Glioma images. a. T2 FLAIR image; b. DWl; c. ADC map; d. T1WI + C; e. CEST MTR map and T2 FLAIR image fusion: The red arrow area is shown as lesion-parenchymal areas, The white arrow area is shown as edema areas and The pink arrow area is shown as non-focus areas; $\mathbf{f}$. CEST MTR map and T1WI + C fusion: The red arrow area is shown as lesion-parenchymal areas, The white arrow area is shown as edema areas and The pink arrow area is shown as non-focus areas

images showed a weak intra-tumor signal and were correlated with the blood supply artery. Cases of brain metastases and gliomas with intratumoral hemorrhage have been excluded by SWI images to avoid affecting the results of CEST MTR $[7,8]$.

Using ASL with PLD $=2.0$, improvement in bloodbrain barrier permeability and the loss of cerebral blood flow regulation can be determined during the evolution of brain metastases and gliomas [9].
The role of 3.0 T MR CEST

In the experiment, T1WI, T2WI, T2 FLAIR, T1WI + C, DWI, SWI, and ASL of 3.0 T MRI was used for verification and observation of the metastatic tumor, glioma, and normal groups. Using the GRE-EPI-CEST sequence of ASSET technology, the $3.5 \mathrm{ppm}$ (MTR) map was obtained by APT software with a quantitative analysis of the corresponding region. The CEST technique and clinical application value of $3.0 \mathrm{~T}$ magnetic resonance lung

Table 1 One-way ANOVA variance test for MTR values in corresponding groups

\begin{tabular}{|c|c|c|c|c|c|c|}
\hline \multirow[t]{2}{*}{ Groups } & \multirow[t]{2}{*}{ Number of cases } & the lesion parenchymal areas & the edema areas & \multirow[t]{2}{*}{ the non focal areas } & \multirow[b]{2}{*}{$F$ value } & \multirow[b]{2}{*}{$P$ value } \\
\hline & & \multicolumn{2}{|c|}{ MTR's Mean \pm standard deviation(\%) } & & & \\
\hline A & 20 & $1.07 \pm 0.22$ & $1.04 \pm 0.23$ & $1.06 \pm 0.24$ & 0.074 & 0.929 \\
\hline B & 26 & $3.29 \pm 1.14$ & $1.28 \pm 0.36$ & $1.26 \pm 0.31$ & 75.219 & 0.000 \\
\hline C & 15 & $6.29 \pm 1.58$ & $2.87 \pm 0.65$ & $1.03 \pm 0.30$ & 99.167 & 0.000 \\
\hline F value & & 98.741 & 87.420 & 4.160 & & \\
\hline$P$ value & & 0.000 & 0.000 & 0.002 & & \\
\hline
\end{tabular}

$P<0.05$, there is a statistical difference

A: normal group; B: metastatic tumor group; C: glioma group 
Table 2 Independent sample t-test of MTR values in corresponding regions between groups

\begin{tabular}{|c|c|c|c|c|c|c|c|c|c|c|c|c|}
\hline \multirow[t]{3}{*}{ Compared } & \multicolumn{4}{|c|}{ the lesion parenchymal areas } & \multicolumn{4}{|c|}{ the edema areas } & \multicolumn{4}{|c|}{ the non focal areas } \\
\hline & \multicolumn{2}{|c|}{ Levene's Test } & \multicolumn{2}{|l|}{ t test } & \multicolumn{2}{|c|}{ Levene's Test } & \multicolumn{2}{|l|}{ t test } & \multicolumn{2}{|c|}{ Levene's Test } & \multicolumn{2}{|l|}{ t test } \\
\hline & F value & $P$ value & $\mathrm{t}$ value & $P$ value & F value & $P$ value & $\mathrm{t}$ value & $P$ value & F value & $P$ value & $\mathrm{t}$ value & $P$ valu \\
\hline normal group \& metastatic tumor group & 14.315 & 0.000 & -8.550 & 0.000 & 0.597 & 0.445 & -2.394 & 0.024 & 0.743 & 0.394 & -2.403 & 0.021 \\
\hline normal group \& glioma group & 21.706 & 0.000 & -14.642 & 0.000 & 6.538 & 0.015 & -11.651 & 0.000 & 1.209 & 0.279 & 0.405 & 0.689 \\
\hline metastatic tumor group \& glioma group & 1.807 & 0.187 & -6.437 & 0.000 & 2.959 & 0.095 & -8.399 & 0.000 & 0.051 & 0.823 & 2.382 & 0.024 \\
\hline
\end{tabular}

Levene's Test, $P<0.05$, homogeneity of variance; independent sample t-test, $P=<0.05$, statistically significant

cancer brain metastasis were discussed. The aim was to develop a CEST-based non-invasive and accurate molecular imaging MRI research program for lung cancer brain metastases.

The results showed the characteristics of CEST signal in lung cancer brain metastasis. The focal parenchymal and edema areas of the metastatic tumor group were reddish yellow and greenish blue. The MTR value was lower than that of the glioma group, which was higher than that of the normal group. However, the non-lesion areas of the metastatic tumor group were greenish blue. The MTR value was higher than that in the normal and glioma groups. The edema and non-lesion MTR values were similar. Besides, these values were higher than those in the normal group, which were mainly in a substantial region (Figs. 1, 2 and 3) [10, 11].

This is related to the mechanism of brain metastases originating from hematogenous metastasis. Tumor cells migrate with blood in different parts of the body, including parts of the brain where no metastatic lesions have been found. As a result, the MTR value of the metastatic tumor lesions is significantly increased. The value of MTR in the edema and non-lesion areas also increased.

If the brain metastases are followed by hemorrhage, the high MTR value of the parenchymal area and the signal in the focal parenchymal areas are unequal. This is related to the content of oxygenated hemoglobin, the content of deoxyhemoglobin, and the necrosis of tumor parenchyma at different periods after hemorrhage. It can be confirmed by conventional sequences combined with SWI and ASL. When SWI showed old bleeding with a significantly low signal, the value of MTR increased by more than $80 \%$. Although the parenchymal areas are all increased, there are wide differences between individuals and regions, which may be due to the coexistence of new and old hemorrhage in the metastatic tumors and tumor necrosis $[7,8]$. CEST can detect blood products (deoxyhemoglobin, methemoglobin, ferritin, and hemosiderin) that cause hemorrhage and a significant increase in MTR. It may also be linked to changes in tissue $\mathrm{pH}$ during the development of metastatic brain tumors, which require further verification of the results. To avoid the interference of bleeding, the cases of intratumoral bleeding were not included in the data of this group.

PTBE will make the tumor occupying effect more apparent, further increase intracranial pressure, and exacerbate clinical symptoms. In MRI, the signal of edema around the brain metastases tumor varies. This is not only linked to macroscopic factors such as nervous system sensitivity, brain metastases position, and the degree of malignancy, but also VEGF and its receptors, AQP-4, MMP-9, IL-6, HIF-1a and other molecular factors $[5,12]$. In this data, the results of VEGF and AQP-4 showed high levels of expression. Particularly in cases of peritumoral edema, AQP-4 was highly expressed in brain tissue around metastatic tumors of the brain, not in metastatic tumors. It explains why the MTR value of CEST in brain metastases is increased and the MTR value in the edema and non-focal areas is more pronounced in cases of bleeding, which may also promote angiogenesis with VEGF. This refers to the causes such as the destruction of the blood-brain barrier. The edema around the tumor is linked to the degree of malignancy of the tumor. The edema around the tumor caused by the malignant tumor which breaks the bloodbrain barrier is mainly angiogenic. The edema primarily invades the white matter of the brain and the fluid with a

Table 3 Independent sample t-test of MTR values between corresponding groups in each group

\begin{tabular}{|c|c|c|c|c|c|c|c|c|}
\hline \multirow[t]{3}{*}{ Compared } & \multicolumn{4}{|c|}{ metastatic tumor group } & \multicolumn{4}{|c|}{ glioma group } \\
\hline & \multicolumn{2}{|c|}{ Levene's Test } & \multicolumn{2}{|l|}{ t test } & \multicolumn{2}{|c|}{ Levene's Test } & \multicolumn{2}{|l|}{ t test } \\
\hline & F value & $P$ value & t value & $P$ value & F value & $P$ value & t value & $P$ value \\
\hline lesion parenchymal areas\& edema areas & 10.107 & 0.003 & 7.207 & 0.000 & 7.252 & 0.012 & 7.748 & 0.000 \\
\hline lesion parenchymal areas\& non focal areas & 15.738 & 0.000 & 8.762 & 0.000 & 14.365 & 0.001 & 12.672 & 0.000 \\
\hline the edema areas\& non focal areas & 0.066 & 0.798 & 0.188 & 0.852 & 3.153 & 0.087 & 9.926 & 0.000 \\
\hline
\end{tabular}

Levene's Test, $P<0.05$, homogeneity of variance; independent sample t-test, $P=<0.05$, statistically significant 
small amount of protein is affected by the damage of the blood-brain barrier and accumulates around the tumor. The degree and magnitude of the edema around the tumor are related to the structure and characteristics of the brain tissue itself. Edema in the cortex, basal ganglia, and thalamus, for example, is not easy to develop. The edema in the white matter region is more noticeable, but not in the brain stem. In this data set, it was also confirmed that 8 cases of edema-free brain metastases occurred in the basal ganglia and thalamus.

The MTR values in the focal parenchymal areas of the glioma group were higher than that of the metastatic tumor group. In contrast, the parenchyma and edema lesion were red, reddish yellow, and their border was undefined. The non-lesion area was greenish blue, not unique from the normal non-incremental group.

The MTR values in the focal parenchymal areas of the metastatic tumor group were higher than those in the edema area and the non-focal area. The MTR values in the edema and non-lesion areas were identical, slightly higher than normal.

In this study, CEST primarily illustrated not only the metabolism of free protein and peptide molecules, but also the behavior of the tumor and its development from the molecular level [13-19]. In the edema areas of the glioma group, the MTR values are increased, consistent with the tumor surrounding the brain and the underlying edema of the tumor. Nevertheless, brain metastases do not penetrate, but compression occurs, the secondary edema of brain metastases, which varies from the initial brain tumors. CEST has certain benefits for the flexibility and precise presentation of the anatomical structure. Abundant free protein or polypeptide molecules can be visually detected in brain metastases. Furthermore, early diagnosis and thorough assessment of the extent of brain metastases and its surrounding anatomical structure $[13,18-21]$ are critical for its treatment and prognosis.

\section{Case selection principles and limitations}

As a structural basis of life, proteins are closely linked to various forms of life activity. Proteins account for between 16 and $20 \%$ of body weight and their amide proton content is high [13, 18-21]. The lung cancer brain metastasis and high-grade gliomas in this research have abnormal metabolism of intracellular proteins and peptides in their disease course. This is the material basis of CEST effect in APT imaging, which is used to differentiate between parenchymal and necrotic regions, surrounding edema area, etc.

Brain metastases account for 10 to $15 \%$ of intracranial tumors and lung cancer brain metastases account for $30-40 \%$ of intracranial tumors [4]. This is also the reason why the experimental data are included in the above-described diseases.
The purpose of this experiment was to test the effect of CEST imaging. All identified brain metastases had arisen owing to lung cancer. Since the brain metastases originated from different types of lung cancer and were of different degrees, the edema and edema size were not analyzed. This, therefore, introduces the limitations of the analysis and will further refine the research in future.

At present, there are some cases of poor signal-tonoise ratio and unsatisfactory CEST image quality contrast. Combined with conventional sequence and improved examination, a certain degree of registration and correction is useful for identification and distinction of lesions, and for the study of brain metastases. The regional signal and its MTR quality can be differentiated between lesion parenchyma, necrosis, and peripheral edema (Figs. 2, 3).

The experimental group will further collect data, expand the range of disease types, randomize data, and analyze the severity and responsiveness of the disease. Simultaneously, we will perform an in-depth study of the fast-APT imaging sequences and seek to use the fast imaging sequence software package to reduce the set of interference signals generated by the EPI sequence, perform intelligent CEST-APT imaging, and provide intelligent and accurate molecular imaging diagnosis information for brain disease research.

\section{Conclusions}

The CEST sequence and APT software using ASSET and GRE-EPI techniques obtain pseudo-color images and reflect protein metabolism. The metastatic and edema areas of the metastatic tumor group were reddish yellow and greenish blue. The level of MTR was lower than that of the glioma group, which was higher than that of the normal group. The non-focal area was greenish blue and the quality of the MTR was higher than that of the glioma and the normal groups.

Combined with the sequence of sweeping, enhancement, DWI/DKI, SWI, ASL/PWI, and MRS, the MTR image resolution and MTR value of CEST $3.5 \mathrm{ppm}$ acyl protons can be used to track the distribution and metabolic changes of brain metastases and conduct early diagnosis of brain metastases and assessment of the outcome of molecular imaging of lesions.

Of course, the clinical application of CEST technology is still at the research stage and further development is required. It is assumed that the importance of its clinical application will become increasingly comprehensive with the advancement of science.

\section{Abbreviations}

APT: Amide proton transfer; AQP: Aquaporin; ASL: Arterial spin labeling; ASSET: Array spatial sensitivity encoding technique; CEST: Chemical exchange saturation transfer; DWI: Diffusion-weighted imaging; FOV: Field of view; FSE: Fast spin-echo; GRE EPI: Gradient recalled echo echoplanar imaging; HIF- 
1a: Hypoxia inducible factor-1a; IL: Interleukin; MMP: Matrix metalloproteinase; MRI: Magnetic resonance imaging; MTR: Magnetization transfer ratio; OAx: Oblique axis; ppm: parts per million; RF: Radiofrequency; ROI: Region of interest; SE: Spin echo; SNR: Signal to noise ratio; SWI: Susceptibility-weighted imaging; T: Tesla; $T_{1} W I+C$ : Enhanced examination (contrast) of $\mathrm{T}_{1}$-weighted imaging; $\mathrm{T}_{1} \mathrm{Wl}: \mathrm{T}_{1}$-weighted imaging; $T_{2}$ FLAIR: $T_{2}$ fluid attenuated inversion recovery; $T_{2} W I: T_{2}$ weighted imaging; TE: Echo time; TR: Repetition time; VEGF: Vascular endothelial growth factor

\section{Acknowledgments}

The authors would like to thank everybody that contributed to this work.

\section{Authors' contributions}

YY designed the entire experimental protocol. As mentors, SD and GG guided the experimental process. Data collection and data analysis were performed by $Y Y, X Q, Y H, G Y, G G$, and $S D$. $X Q$ is a member of the research team and sends his students to collect analytical data. All authors have been involved in drafting and revising the manuscript and approved the final version to be published. All authors read and approved the final manuscript.

\section{Authors' information}

Author Names and Degrees: Yonggui Yang, MM. Xiaobo Qu, PhD. Yihui Huang. Khan Afsar. Gen Yan, PhD. MD. Gang Guo, MD. Shaoyin Duan, PhD. MD.

\section{Funding}

This work was supported by a Planned Project Grant from the Science and Technology Bureau of Xiamen (Grant No. 3502Z20144052), a grant from the Natural Science Foundation of Fujian Province of China (Grant No. 2018 J06018), and grant from the Fundamental Research Funds for the Central Universities (Grant No. 20720180056). The first funding body had a role in the design of the study, collection, analysis, and interpretation of data, and in writing the manuscript. The two remaining funding bodies had roles in the study design, interpretation of data, and in writing the manuscript.

\section{Availability of data and materials}

All data generated or analyzed during this study are included in this published article.

\section{Ethics approval and consent to participate}

The research project was reviewed and approved by the Medical Ethics Committee of The Second Affiliated Hospital of Xiamen Medical College (Approval No: 2014004). All subjects were informed of the examination and signed an informed consent before the examination. Written informed consent was obtained from all participants.

\section{Consent for publication}

All presentations of case reports had consent for publication.

\section{Competing interests}

The authors declare that they have no competing interests.

\section{Author details}

'Department of Radiology, The Second Affiliated Hospital of Xiamen Medical College, Xiamen, Fujian Province, China. ${ }^{2}$ Department of Electronic Science, Fujian Provincial Key Laboratory of Plasma and Magnetic Resonance, Xiamen University, Xiamen, Fujian Province, China. ${ }^{3}$ Department of Radiology,

Zhongshan Hospital Xiamen University, Xiamen, Fujian Province, China.

Received: 24 June 2019 Accepted: 8 December 2019

Published online: 13 January 2020

\section{References}

1. Sun PZ, Sorensen AG. Imaging $\mathrm{pH}$ using the chemical exchange saturation transfer (CEST)MRl: correction of concomitant RF irradiation effects to quantify CESTMRI for chemical exchange rate and pH. Magn Reson Med. 2008;60:390-7

2. Ward KM, Aletras AH, Balaban RS. A new class of contrast agents for MRI based on proton chemical exchange dependent saturation transfer (CEST). J Magn Reson. 2000;143:79-87.
3. Yang YG, Shen ZW, Wu RH, et al. Research of chemical exchange saturation transfer in brain. Chin J Magn Reson Imaging. 2016;7:81-4.

4. MaQ MH, Zhang S, et al. MR findings of brain metastases from different primary tumors. Chinese J CT MRI. 2018;16:30-2.

5. Gao J, Liu YH, Yan L, et al. Research progress on peritumoral edema-related molecular biology factors of the metastasis tumor of brain. Chin J Clin Oncol. 2014:41:534-7.

6. Wang E, Wu Y, Cheung JS, et al. pH imaging reveals worsened tissue acidification in diffusion kurtosis lesion than the kurtosis/diffusion lesion mismatch in an animal model of acute stroke. J Cereb Blood Flow Metab. 2017:37:3325-33.

7. Liang S, Zhang FX. The value of SWI in diagnosis of brain metastases with lung cancer. J Pract Med Imaging. 2017;18:7-9.

8. Li L, Liu SM, Li GQ, et al. Susceptibility weighted imaging in thrombolytic therapy of acute ischemic stroke. Chin Med J. 2017;130:2489-97.

9. Mirasol RV, Bokkers RPH, Hernandez DA, et al. Assessing reperfusion with whole-brain arterial spin labeling. Stroke. 2014;45:456-61.

10. Yang YG, Guo G. The application of different NEX amide proton transfer imaging in brain tumors on 1.5 T MRI scanner. Chin J Magn Reson Imaging. 2017:8:923-8

11. Yang $Y G$, Chen $Z$, Cai CB, et al. Factors affecting chemical exchange saturation transfer imaging on $1.5 \mathrm{~T}$ clinical MRI scanners. Chinese J Magn Reson Imaging. 2017;34:275-82.

12. Dong $\mathrm{P}$, Wang $\mathrm{B}$, Sun $\mathrm{XH}$, et al. Factors of the edema size of intracranial metastases from lung cancer. Chin J Med Imaging Technol. 2006;22:57-60.

13. Zhou J, Wilson DA, Sun PZ, et al. Quantitative description of proton exchange processes between water and endogenous and exogenous agents for WEX, CEST and APT experiments. Magn Reson Med. 2004;51:945-52.

14. van Zijl PC, Yadav NN. Chemical exchange saturation transfer (CEST): What is in a name and what isn't? Magn Reson Med. 2011;65:927-48.

15. Zhou J, Lal B, Wilson DA, et al. Amide proton transfer (APT) contrast for imaging of brain tumors. Magn Reson Med. 2003;50:1120-6.

16. McConnell HM. Reaction rates by nuclear magnetic resonance. J Chem Phys. 1958;28:430.

17. Mori S, Eleff SM, Pilatus U, et al. Proton NMR spectroscopy of solventsaturable resonances: a new approach to study pH effects in situ. Magn Reson Med. 1998:40:36-42.

18. Sun PZ, Longo DL, Hu W, et al. Quantification of iopamidol multi-site chemical exchange properties forratiometric chemical exchange saturation transfer (CEST) imaging of pH. Phys Med Biol. 2014;59:4493-504.

19. Sun PZ, Wang Y, Dai Z, et al. Quantitativechemical exchange saturation transfer (qCEST) MRI--RF spillover effect-correctedomega plot forsimultaneous determination of labile proton fraction ratio and exchange rate. Contrast Med Mol Imaging. 2014;9:268-75.

20. Sun PZ, Wang E, Cheung JS, et al. Simulation and optimization of pulsed radio frequency (RF) irradiation scheme for chemical exchange saturation transfer (CEST) MRI - demonstration of pH-weighted pulsed-amide proton CEST MRI in an animal model of acute cerebral ischemia. Magn ResonMed. 2011;66:1042-8.

21. Sun PZ, Wang E, Cheung JS. Imaging acute ischemic tissue acidosis with pH-sensitive endogenous amide proton transfer (APT) MRI-Correction of tissue relaxation and concomitant RF irradiation effects toward mapping quantitative cerebral tissue pH. Neuroimage. 2012;60:1-6.

\section{Publisher's Note}

Springer Nature remains neutral with regard to jurisdictional claims in published maps and institutional affiliations.

\section{Ready to submit your research? Choose BMC and benefit from}

- fast, convenient online submission

- thorough peer review by experienced researchers in your field

- rapid publication on acceptance

- support for research data, including large and complex data types

- gold Open Access which fosters wider collaboration and increased citations

- maximum visibility for your research: over $100 \mathrm{M}$ website views per year

At BMC, research is always in progress.

Learn more biomedcentral.com/submission 